\title{
Dynamic Supply and Threshold Voltage Scaling for CMOS Digital Circuits Using In-Situ Power Monitor
}

\author{
Nandish Mehta, Student Member, IEEE, and Bharadwaj Amrutur, Member, IEEE
}

\begin{abstract}
A generalized power tracking algorithm that minimizes power consumption of digital circuits by dynamic control of supply voltage and the body bias is proposed. A direct power monitoring scheme is proposed that does not need any replica and hence can sense total power consumed by load circuit across process, voltage, and temperature corners. Design details and performance of power monitor and tracking algorithm are examined by a simulation framework developed using UMC 90-nm CMOS triple well process. The proposed algorithm with direct power monitor achieves a power savings of $42.2 \%$ for activity of 0.02 and $22.4 \%$ for activity of 0.04 . Experimental results from test chip fabricated in AMS $350 \mathrm{~nm}$ process shows power savings of $46.3 \%$ and $65 \%$ for load circuit operating in super threshold and near sub-threshold region, respectively. Measured resolution of power monitor is around $0.25 \mathrm{mV}$ and it has a power overhead of $2.2 \%$ of die power. Issues with loop convergence and design tradeoff for power monitor are also discussed in this paper.
\end{abstract}

Index Terms-CMOS, dynamic voltage and threshold scaling (DVTS), in-situ power monitor, leakage current control, low power, power optimum point, sleep transistor, variable body bias, variable supply voltage, $V_{\mathrm{DD}}-V_{\mathrm{TH}}$ control.

\section{INTRODUCTION}

$\mathbf{P}$ OWER consumption of modern digital integrated CMOS circuits is increasing with each generation and has become a serious design issue. Traditionally, dynamic power management (DPM) is employed at operating system level to adjust the supply voltage for each power state. The supply voltage is conservatively margined to account for process and temperature variations. These voltage margin increases with technology scaling due to larger process variations, rendering DPM less efficient. On the other hand, the hardware approach like dynamic voltage scaling (DVS) allows voltage to be scaled such that the actual delay of the chip instead of worst case delay meets the target performance. This enables more power savings as minimum possible voltage for target performance can be attained. In DVS the supply voltage is adjusted to meet the target delay using an on-chip delay monitor in a hardware feedback loop [1].

Though DVS very well manages the dynamic switching power, with shrinking feature size the static (leakage) power

Manuscript received July 16, 2010; revised November 30, 2010 and February 10, 2011; accepted March 12, 2011. This work was supported in part by grants from MCI, Government of India.

The authors are with the Microelectronics Laboratory, Department of Electrical Communication Engineering, Indian Institute of Science, Bangalore, Karnataka 560012, India (e-mail: nandish@ece.iisc.ernet.in; amrutur@ece.iisc.ernet.in).

Color versions of one or more of the figures in this paper are available online at http://ieeexplore.ieee.org.

Digital Object Identifier 10.1109/TVLSI.2011.2132765 has increased exponentially which it cannot control. Particularly, at low activity levels, leakage power is dominant. Dynamic voltage and threshold scaling (DVTS) manages both dynamic and leakage power by adjusting supply voltage $\left(V_{\mathrm{DD}}\right)$ and body bias voltage $\left(V_{B S}\right)$. In digital circuits designed below $0.1 \mu \mathrm{m}$, total power at any given performance can be optimized by DVTS [2]. DVTS has been successfully proven on silicon for low power high speed applications [3]-[5]. The optimum power point varies strongly with activity and temperature. Hence, it is necessary to locate optimum $\left\{V_{\mathrm{DD}}, V_{B S}\right\}$ automatically [3], [4], [6]. To locate the optimum point, unlike in DVS, DVTS needs information about total power consumption of the circuit. In [3] a DVTS closed-loop system is designed, where a feedback loop is used to maintain the ratio of $I_{S W} / I_{\text {Leak }}$ for which power is optimum. Power information is obtained from replica circuits that mimic leakage and switching power. The major drawback of replica circuits is that they cannot track variation in power due to activity and temperature. Also designing replica circuits to mimic power dissipation of large circuit blocks is challenging due to a diversity of gate types and increased random process variations in deep sub-micrometer (DSM) process nodes. Technique to estimate the dynamic power by monitoring activity directly from the load circuit is proposed in [7] and [8]. However, for DVTS leakage power also needs to be measured. Hence, it is desirable to measure power directly from the actual circuit. One direct measurement approach is to measure the voltage drop across series sense resistor and estimate the total current drawn. The power consumed is obtained by multiplying estimated current with the supply voltage. The resistance embedded in core power plane can be used as sense resistor [9]. For designs with power gating, the power gating sleep transistors can be used as sense resistors as described in this paper. Absolute power measurements as in [9] requires two high resolution analog-to-digital converters (ADCs), a multiplier and a calibration hardware to know the sense resistor value.

In this paper a DVTS algorithm is described which needs relative power values between two measurement iterations. This differential operation reduces the measurement area and power overhead significantly. Further, an in-situ power monitoring technique is proposed which enables tracking of power with activity and/or temperature variations. The in-situ operation of power monitor permits DVTS algorithm to optimize the power regardless of the operating region (super or sub-threshold) of load circuit. The proposed DVTS system is validated by a AMS $350 \mathrm{~nm}$ CMOS test chip.

Section II explains DVTS system and the control algorithm. Section III deals with the design and implementation of the 


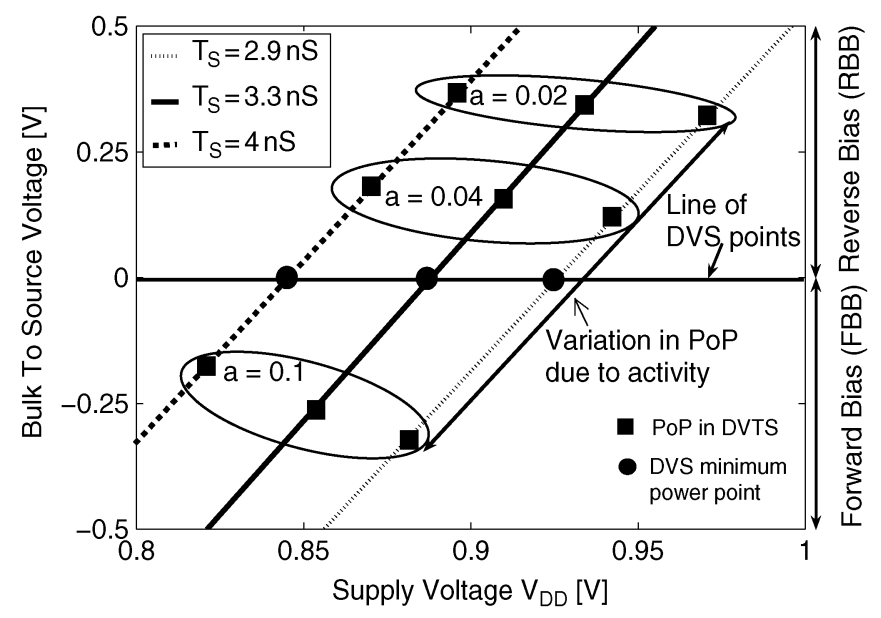

Fig. 1. $\left\{V_{\mathrm{DD}}, V_{B S}\right\}$ design space for ring oscillator mimicking Type-III load circuit. $T_{S}$ is the constant target performance.

TABLE I

Percentage of Power SAVINGS By DVTS Over DVS

\begin{tabular}{|c|c|c|c|c|c|c|c|c|c|}
\cline { 2 - 10 } \multicolumn{1}{c|}{} & \multicolumn{2}{c|}{$\begin{array}{l}\text { Type I: Microprocessor } \\
\text { Logic Depth }=11\end{array}$} & \multicolumn{3}{c|}{$\begin{array}{c}\text { Type II: ASIC/ASIP } \\
\text { Logic Depth }=31\end{array}$} & \multicolumn{3}{c|}{$\begin{array}{c}\text { Type III: Moderate } T_{S} \\
\text { Logic Depth }=101\end{array}$} \\
\hline Activity & $5^{\circ} \mathrm{C}$ & $75^{\circ} \mathrm{C}$ & $125^{\circ} \mathrm{C}$ & $5^{\circ} \mathrm{C}$ & $75^{\circ} \mathrm{C}$ & $125^{\circ} \mathrm{C}$ & $5^{\circ} \mathrm{C}$ & $75^{\circ} \mathrm{C}$ & $125^{\circ} \mathrm{C}$ \\
\hline 0.001 & 7.14 & 14.75 & 26.6 & 17.8 & 20.4 & 24.6 & 21.5 & 23.4 & 27.3 \\
\hline 0.01 & 4.3 & 7.7 & 22.2 & 10.7 & 13.8 & 22.7 & 20.7 & 21.6 & 25.9 \\
\hline 0.05 & 4 & 6.2 & 9.3 & 4 & 4.6 & 13.7 & 6.7 & 13.6 & 22.12 \\
\hline 0.1 & 2 & 3.3 & 5.5 & 2.3 & 2 & 8.8 & 4.6 & 9.5 & 18.1 \\
\hline
\end{tabular}

power monitor. Section IV discusses concerns while implementing DVTS with in-situ power monitor. Section V presents measured result of test chip. Section VI concludes this work with possible future improvements.

\section{PROPOSED DVTS SYSTEM}

\section{A. DVTS Versus DVS}

To quantify the power savings achieved by DVTS over DVS, ring oscillators with different logic depth to mimic a high speed microprocessor, a high performance ASIC and a moderate performance ASIC are designed. These oscillators are simulated in HSPICE using UMC $90 \mathrm{~nm}$ CMOS process. The inclined lines in Fig. 1 are the constant performance curves. These curves are the locus of $\left\{V_{\mathrm{DD}}, V_{B S}\right\}$ points for which performance remains same but power varies. Out of all these points power is minimum only at the power optimum point (PoP). $V_{B S}$ is the body bias to control the threshold voltage of the nMOS and pMOS transistor. ${ }^{1}$

At small activity and/or temperature leakage power is dominant which is reduced by increasing $V_{B S}$. To maintain constant performance, $V_{\mathrm{DD}}$ is also increased. Though the dynamic power increases, net total power reduces. Whereas, at very high activity, the dynamic power dominates the total power which is reduced by reducing $V_{\mathrm{DD}}$. The performance degradation is compensated by reducing $V_{B S}$. Unlike in DVS where $V_{\mathrm{DD}}$ is set to meet target performance, in DVTS combination of $\left\{V_{\mathrm{DD}}, V_{B S}\right\}$

\footnotetext{
${ }^{1} V_{B S}$ is considered with pMOS transistor as reference. It has opposite polarity and same magnitude for nMOS transistors.
}

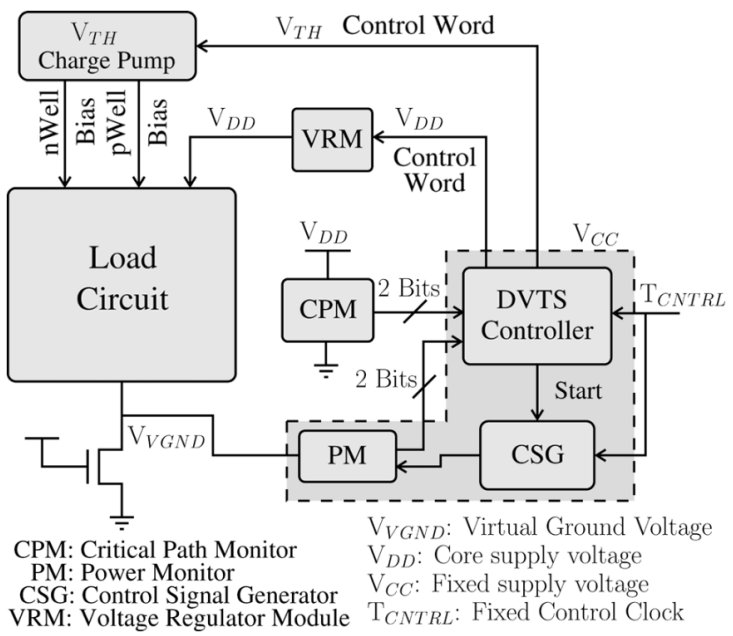

Fig. 2. Proposed DVTS system block diagram.

together determines performance and power which improves power saving at higher activity. Thus, under DVTS control the load circuit always operates at PoP by moving diagonally along the constant performance curve by adjusting $V_{\mathrm{DD}}$ and $V_{B S}$.

Table I summarizes the power savings achieved by DVTS over DVS under steady state condition with varying activity and temperature. DVTS offers considerable power savings compared to DVS when leakage power is a large fraction of total power. The power savings increase with increasing logic depth. The moderate performance application-specific integrated circuit (ASIC) with long logic depth at low activity and/or high temperatures gain the most with DVTS. Thus in future DSM nodes with larger sub-threshold leakage, DVTS will be an attractive option to reduce power.

\section{B. DVTS Controller}

Fig. 2 shows block diagram of close loop DVTS system with in-situ power monitor. DVTS controller implements the proposed DVTS algorithm and controls the supply voltage regulator and well bias charge pump. The drain of sleep transistor acts as a virtual ground node for the load circuit. The power monitor processes virtual ground voltage $\left(V_{\mathrm{VGND}}\right)$ to generate a 2 bit output that gives information on total power consumed by the load. Control signal generator (CSG) generates control signals for power monitor when START signal is asserted. The delay monitor measures whether performance of load circuit meets the target performance within tolerance limit. Delay monitor is implemented with a critical path replica circuit [10]. The proposed DVTS system is suited for load circuits where the rate of change in activity is gradual. The maximum rate of change of activity that can be supported is discussed in Section IV-A.

\section{DVTS Algorithm}

Fig. 3 shows the flow chart of the proposed DVTS algorithm. The algorithm first sets the supply voltage to meet target performance, then adjust well potential to achieve minimum power. nWell bias and pWell bias are always tuned by same amount and hence DVTS control loop is essentially a 2-D control loop. To avoid oscillations, $V_{\mathrm{DD}}$ loop and $V_{B S}$ loop are decoupled by tuning them independently [4]. Performance is said to have met 


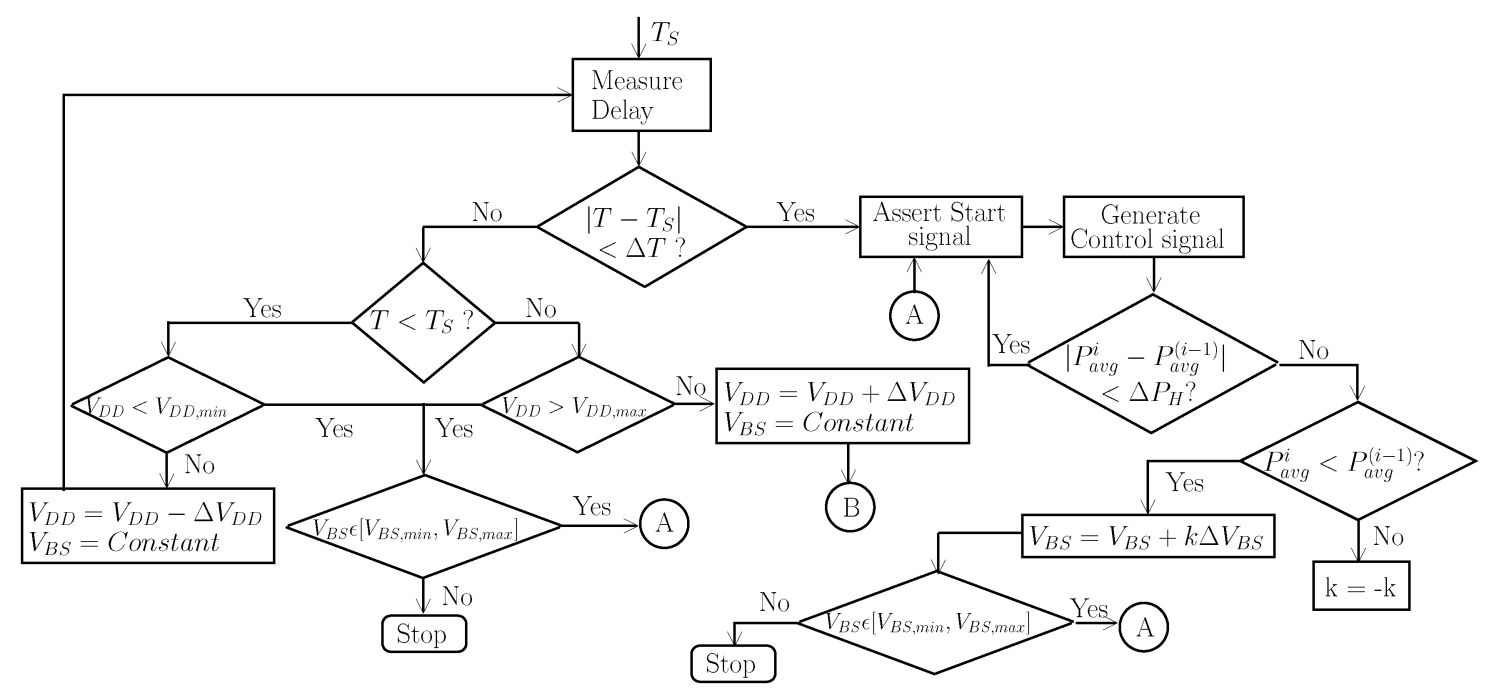

Fig. 3. Flow Chart of DVTS algorithm. $V_{\mathrm{DD}, \max }$ to $V_{\mathrm{DD} \text {, min }}$ and $V_{B S, \max }$ to $V_{B S \text {, min }}$ is allowable range for $V_{\mathrm{DD}}$ and $V_{B S}$, respectively.

when current performance $T$ is within tolerance window $\Delta T$ of target performance $T_{S}$. It is achieved by increasing or decreasing $V_{\mathrm{DD}}$ by step size of $\Delta V_{\mathrm{DD}}$. Power monitor is triggered once performance is met. Note that minimizing power at fixed target performance is equivalent to minimizing energy.

Power monitor computes difference between power consumed in current step $\left(P_{\text {avg }}^{i}\right)$ and the power consumed in previous step $\left(P_{\text {avg }}^{(i-1)}\right)$. If the difference is below the power threshold level $\Delta P_{H}$, power monitor observes no change in power and algorithm converges to PoP. But if the difference is greater than $\Delta P_{H}$ then based on its sign, power monitor decides whether power is increasing or decreasing. The algorithm steps well bias $V_{B S}$ by step size $\Delta V_{B S}$ based on decision of power monitor. After every $V_{B S}$ step performance is measured as it might have got violated due to change in $V_{T H}$. Once algorithm converges to PoP it reads power monitor continuously to detect any change in power due to change in activity and/or temperature. The algorithm reinitiates itself if a power change greater than $\Delta P_{H}$ is observed.

$T_{S}$ is set via software application like in any other DVS technique. But once target frequency is set, the PoP variation is tracked automatically (without software intervention) with activity and temperature. Thus, the software policies for run-time DVS can be reused [11]. Fig. 4(a) shows behavior of DVTS algorithm while tracking PoP and Fig. 4(b) compares the total power of load circuit under no DVTS, ideal DVTS and DVTS with finite step size. Suppose, the activity changes to 0.04 at point A then the DVTS loop initiates. Setting $V_{B S}$ to maximum forward bias [see Fig. 4(a)] avoids load circuit entering into nonfunctional point due to reduced overdrive. Once the algorithm meets constant performance curve at point $\mathrm{B}$, it updates the direction of stepping $V_{B S}$. This direction is also updated every time supply voltage is modified which leads to ping-pong steps shown in Fig. 4(a). After loop settles at point C, the DVTS loop enters continuous power monitor reading mode.

Suppose after reading the power monitor for time $T_{C}$ [see Fig. 4(b)], change in power greater than $\Delta P_{H}$ due to change in activity from 0.04 to 0.02 is noticed. Then the loop reinitiates

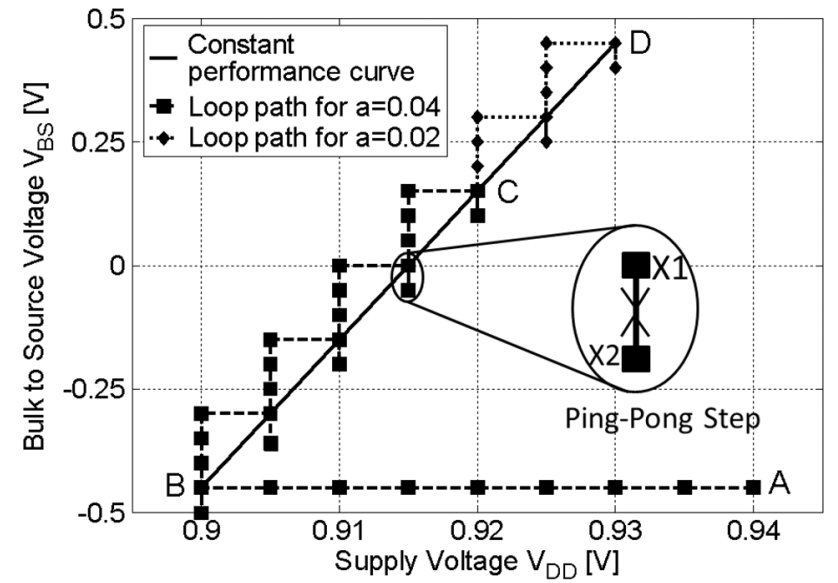

(a)

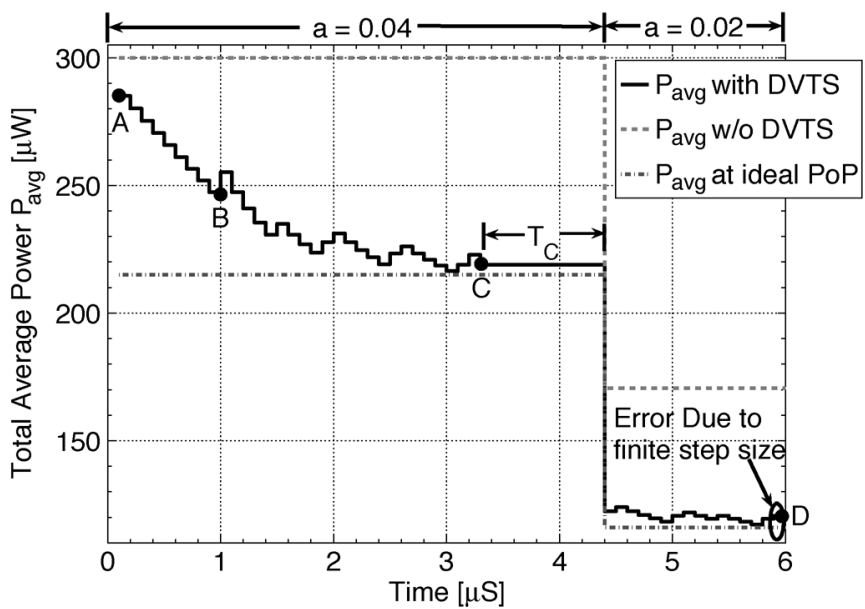

(b)

Fig. 4. (a) DVTS loop convergence with two different activity factors (b) power under DVTS with $\Delta V_{\mathrm{DD}}=5 \mathrm{mV}$ and $\Delta V_{B S}=50 \mathrm{mV}$.

itself [see Fig. 4(a)] to search for new PoP. As point-C itself lies on the constant performance curve, the loop no longer hunts for it. The loop settles at the new PoP at point-D and again enters in continuous power monitor reading state. The DVTS algorithm 

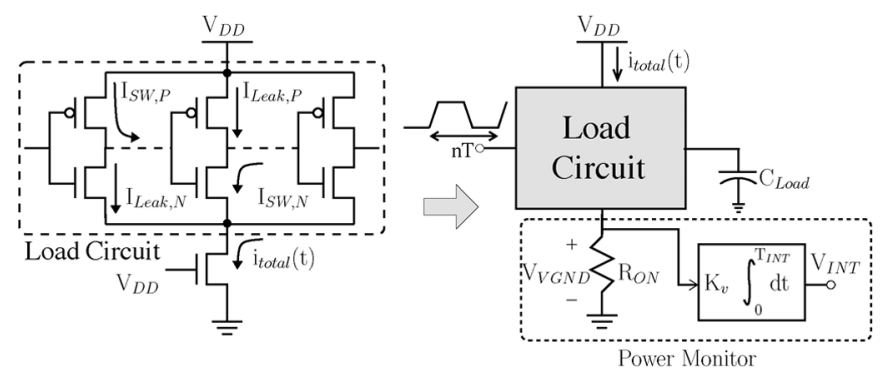

Fig. 5. Equivalent circuit of invasive power monitor with sleep transistor as power sensor.

has an overhead of approximately 600 gates when synthesized using Faraday standard cells for $90 \mathrm{~nm}$ CMOS.

\section{DifFERENCE POWER MONITOR DESIGN}

The voltage drop across sleep transistor is used by the power monitor to measure the total power consumption [12]. Sizing of sleep transistor with low performance degradation due to voltage drop is well known and it is assumed for this work.

\section{A. Theoretical Background of Power Monitor}

In active mode the sleep transistor works in linear region and hence its ON resistance can be modeled by $R_{\mathrm{ON}}$. As shown in Fig. 5 , if $i_{\text {Total }}$ is the total current demand of the load circuit than a voltage drop of $V_{\mathrm{VGND}}$ develops across $R_{\mathrm{ON}}$. The output of the integrator with gain $K_{v}$ is

$$
\begin{aligned}
V_{\mathrm{INT}} & =K_{v} \int_{0}^{T_{\mathrm{INT}}} V_{\mathrm{VGND}}(t) d t=K_{v} R_{\mathrm{ON}} \int_{0}^{T_{\mathrm{INT}}} i_{\mathrm{Total}}(t) d t \\
& =K_{v} R_{\mathrm{ON}} I_{\mathrm{avg}} T_{\mathrm{INT}} .
\end{aligned}
$$

Relation between average power $P_{\text {avg }}$ and $V_{\text {INT }}$ can be obtained by using $P_{\mathrm{avg}}=V_{\mathrm{DD}} \cdot I_{\mathrm{avg}}$ in the equation above

$$
P_{\mathrm{avg}}=\left(\frac{V_{\mathrm{DD}}}{K_{v} T_{\mathrm{INT}} R_{\mathrm{ON}}}\right) V_{\mathrm{INT}} .
$$

The average power is proportional to $V_{\mathrm{DD}} \cdot V_{\mathrm{INT}}$. In order to remove $V_{\mathrm{DD}}$ term from (2), one solution is to make integration time proportional to $V_{\mathrm{DD}}$. This is achieved by dividing $T_{\mathrm{CNTRL}}$ by a bit programmable counter. Thus, the relation between $T_{\mathrm{INT}}$ and $V_{\mathrm{DD}}$ can be written as

$$
T_{\mathrm{INT}}=N T_{\mathrm{CNTRL}}=k_{d} V_{\mathrm{DD}} T_{\mathrm{CNTRL}}
$$

where $N=k_{d} V_{\mathrm{DD}}$. Unit of $k_{d}$ is $1 / V$. By (2) and (3)

$$
P_{\mathrm{avg}}=k_{a} V_{\mathrm{INT}} \text { where, } k_{a}=\left(\frac{R_{\mathrm{INT}} C_{\mathrm{INT}}}{k_{d} T_{\mathrm{CNTRL}} R_{\mathrm{ON}}}\right) \text {. }
$$

Thus, $P_{\text {avg }}$ can be measured as a scaled version of $V_{\mathrm{INT}}$. As $k_{a}$ depends on $R_{\mathrm{ON}}$, its precise value is not known and hence (4) cannot measure absolute power. Fig. 6 shows simulation results for actual power consumption and $V_{\mathrm{INT}}$. $\left\{V_{\mathrm{DD} \text {,opt }}, V_{B S \text {,opt }}\right\}$ located by tracking minima in $V_{\mathrm{INT}}$, matches with actual PoP coordinates in $P_{\text {avg }}$. Actual power curve is obtained by SPICE

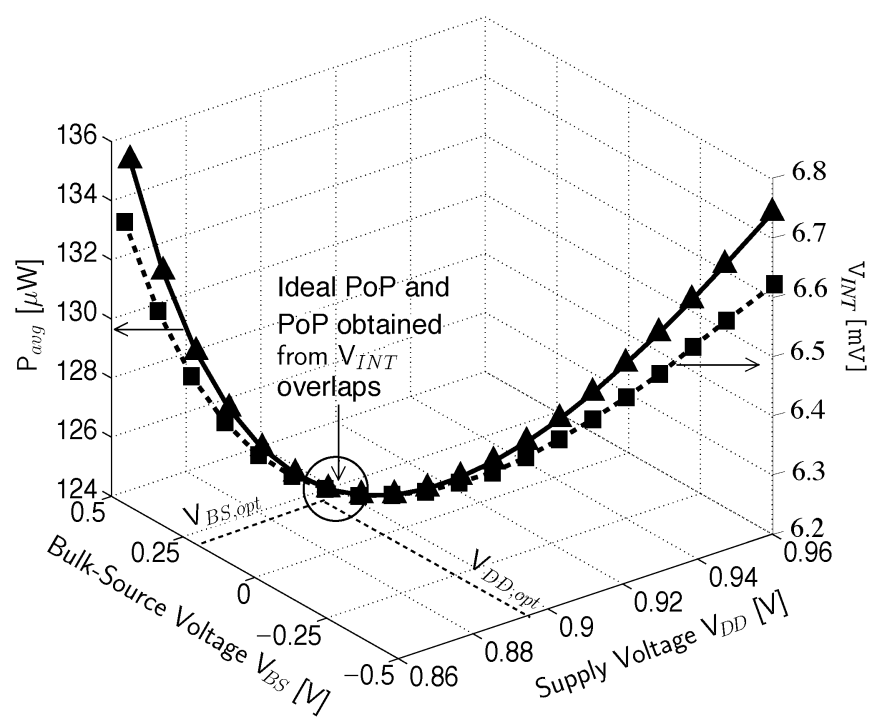

Fig. 6. $V_{\mathrm{DD} \text {,opt }}$ and $V_{B S \text {,opt }}$ for Type-III circuit obtained by locating minima in $V_{\text {INT }}$ and minima in $P_{\text {avg. }}$. Both minima match exactly.

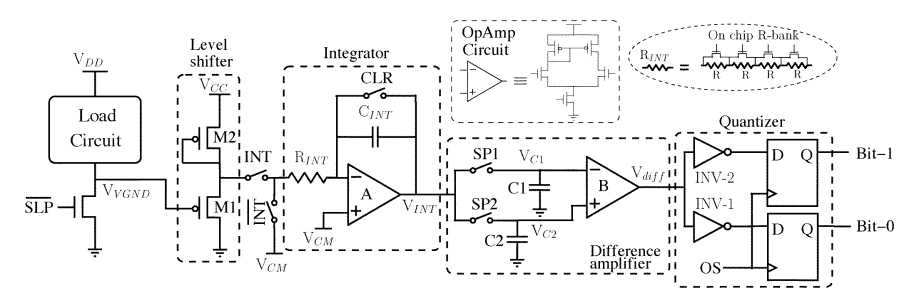

Fig. 7. Circuit diagram of power monitor.

simulations and $V_{\text {INT }}$ curve is obtained by integrating $V_{\text {VGND }}$ using integrator of Fig. 7. The $P_{\text {avg }}$ and $V_{\mathrm{INT}}$ does not match exactly because $k_{a}$ varies from 48.9 to $50 \mathrm{mV} / \mu \mathrm{W}$.

\section{B. Circuit Implementation of Power Monitor}

The power monitor in Fig. 7 is designed in UMC $90 \mathrm{~nm}$ CMOS 1P9M process. Level shifter shifts $V_{\mathrm{VGND}}$ to the input common mode of opamp-A. $R_{\mathrm{INT}}$ is implemented by a resistor bank to have programmable integration time constant. The active Miller integrator is implemented with $R_{\mathrm{INT}}, C_{\mathrm{INT}}$ and opamp-A. Since the DVTS system only needs relative information of increase or decrease in the power rather than accurate measurement of the absolute power, power monitor uses a difference amplifier and a 2 bit quantizer to digitize $V_{\text {INT }}$ instead of an ADC. In addition, this design choice reduces power and area overhead of the power monitor. The output of the difference amplifier with gain $A_{\text {diff }}$ over two consecutive $(i-1)$ th and $i$ th step is given as

$$
\left|V_{\mathrm{diff}}^{i}\right|=A_{\mathrm{diff}}\left(V_{\mathrm{INT}}^{(i-1)}-V_{\mathrm{INT}}^{i}\right)=A_{\mathrm{diff}} \Delta V_{\mathrm{INT}}^{i} .
$$

Output of the quantizer with threshold $\Delta V_{H}$ is set when

$$
V_{\text {diff }}^{i} \geqslant \frac{\Delta V_{H}}{2} \text { where } \Delta V_{H}=V_{T H, \text { High }}-V_{T H, \text { Low }} .
$$

If the difference in power during $(i-1)$ th and $i$ th step is given by $\Delta P^{i}=P_{\mathrm{avg}}^{i}-P_{\mathrm{avg}}^{(i-1)}$ than using (4), (5) and the gain of 

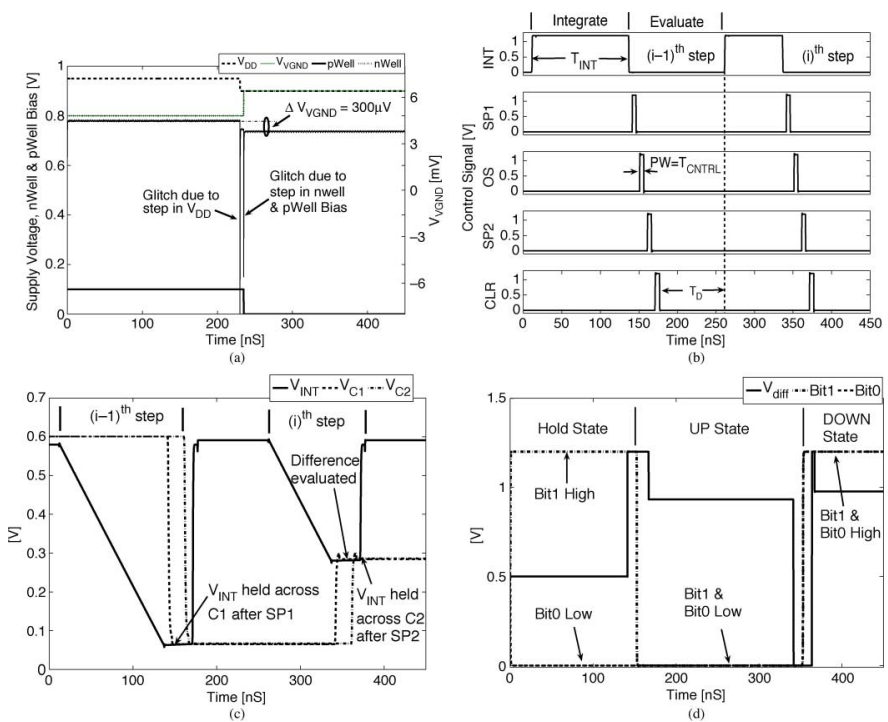

Fig. 8. Simulation waveform at various stages in power monitor circuit for $(i-1)$ th and $i$ th step. (a) Step in $V_{\mathrm{DD}}$ and $V_{B S}$ lines; (b) timing of control signals; (c) output of integrator and voltage across hold capacitors; (d) output of difference amplifier and output latches.

integrator, $A_{\mathrm{INT}}=T_{\mathrm{INT}} /\left(R_{\mathrm{INT}} C_{\mathrm{INT}}\right)$, the power threshold $\Delta P_{H}$ of the power monitor is given as

$$
\Delta P_{H}=\left(\frac{\Delta V_{H}}{2}\right) \frac{R_{\mathrm{INT}} C_{\mathrm{INT}}}{A_{\mathrm{diff}} k_{d} T_{\mathrm{CNTRL}} R_{\mathrm{ON}}} .
$$

The power monitor will indicate an increase in power or UP state if, $\Delta P^{i} \geqslant \Delta P_{H}$ and will indicate decrease in power or a DOWN state if, $\Delta P^{i} \leqslant-\Delta P_{H}$. The power monitor indicates HOLD state when $\Delta P^{i}$ is within the two bounds.

The power monitor operates in two phases, viz. integration phase and evaluation phase [see Fig. 8(b)]. During integration phase INT pulse integrates $V_{\text {VGND }}$ value for $T_{\mathrm{INT}}$ duration. During evaluation phase, the output of the integrator, $V_{\mathrm{INT}}$, is held across $C_{\mathrm{INT}}$. During $(i-1)$ th step $V_{\mathrm{INT}}$ is sampled across $\mathrm{C} 2$ by SP2 pulse [see Fig. 8(c)]. C2 holds the charge until next measurement cycle. During $i$ th step it is compared with $V_{\mathrm{INT}}$ sampled across C1 by SP1 pulse. The result of comparison is latched during OS. CLR pulse removes residual charge left across $C_{\mathrm{INT}}$. The voltage transfer curve (VTC) of inverter based skewed quantizer is shown in Fig. 9. Fig. 8(c) shows the 2 bit output, Bit- 0 and Bit- 1 , of power monitor. Time delay $T_{D}$ in Fig. 8(b) is time required by charge pump to settle to current $V_{B S}$ step values. The total measurement time $T_{\text {meas }}$ is given by

$$
T_{\text {meas }}=T_{\mathrm{INT}}+4 T_{\mathrm{CNTRL}}+T_{D, \mathrm{VBS}} .
$$

$T_{\text {meas }}$ is not constant as $T_{\mathrm{INT}}$ varies with $V_{\mathrm{DD}}$ from $T_{\mathrm{INT}}^{\mathrm{min}}$ to $T_{\mathrm{INT}}^{\max }$. The control signals adds up to $4 T_{\mathrm{CNTRL}}$.

\section{Non-Ideal Effects in Power Monitor}

The input offset voltage of the integrator hampers the dynamic range and it impacts the most when integrator operates with maximum integration time. It can be mitigated by sizing the input pair properly and choosing larger $C_{\mathrm{INT}}$. The input offset voltage, $V_{O S}$, of the difference amplifier reduces the resolution of the power monitor. $V_{O S}$ can be managed by auto-zeroing techniques [13]. The charge injection from switches SP1 and SP2 on hold capacitor appears as common mode signal to difference amplifier and thus gets cancelled to first order. Further, the leakage current also degrades the resolution of the power monitor by discharging the hold capacitor (C2). Error due to leakage current and charge injection results in error voltage $\Delta V$ across the hold capacitors. The change in the gain of the integrator over consecutive integration phase results in residue output offset voltage $\Delta V_{\mathrm{INT} \text {,off }}$. These nonidealities alters the power threshold from (6) to

$$
\begin{aligned}
& \Delta P_{H}=\left[\Delta V_{\mathrm{INT}, \mathrm{off}}+\frac{\left(\frac{\Delta V_{H}}{2}+V_{\mathrm{OS}}\right)}{A_{\mathrm{diff}}}+\Delta V\right] \\
& \times\left(\frac{R_{\mathrm{INT}} C_{\mathrm{INT}}}{k_{d} T_{\mathrm{CNTRL}} R_{\mathrm{ON}}}\right)+\sqrt{2} \sqrt{P_{a}^{2}+\left(\frac{V_{\mathrm{DD}}}{R_{\mathrm{ON}}}\right)^{2} \frac{\overline{v_{n}^{2}}}{T_{\mathrm{INT}} f}} .
\end{aligned}
$$

The activity of load circuit changes due to change in input vectors while power monitor is making its measurement. As explained in the appendix, this activity dependent noise, appears in the power measurement as $P_{a} \cdot \overline{v_{n}}$ is the noise of the power monitor. From (8) it can be seen that by, increasing $T_{\mathrm{INT}}, P_{a}$ and $\overline{v_{n}}$ can be averaged out to improve the power resolution. $P_{a}$ and $\overline{v_{n}}$ are assumed to be independent across two sampling instances resulting in multiplier of $\sqrt{2}$.

\section{SYSTEM UNDER DVTS CONTROL}

\section{A. Timing Overhead of DVTS}

The convergence time of DVTS algorithm depends on its current operation point. If the load circuit operates currently at $\left\{V_{\mathrm{DD}, \mathrm{curr}}, V_{B S, \mathrm{curr}}\right\}$ than the number of steps, $Q$, required in $V_{\mathrm{DD}}$ to reach $\mathrm{PoP}$ is $\left(V_{\mathrm{DD}, \text { curr }}-V_{\mathrm{DD} \text {, opt }}\right) / \Delta V_{\mathrm{DD}}$. The number of steps, $P$, in $V_{B S}$ required to reach $\mathrm{PoP}$ is $\left(V_{B S, \text { curr }}-V_{B S, \text { opt }}\right) / \Delta V_{B S}$. Using (7), the total convergence time, $T_{\text {Total }}$, is given by

$$
T_{\text {Total }}=P\left[4 T_{\mathrm{CNTRL}}+T_{D, \mathrm{VBS}}+T_{\mathrm{INT}}\right]+Q\left[T_{D, \mathrm{VDD}}\right]
$$

where, $T_{D, \mathrm{VBS}}$ and $T_{D, \mathrm{VDD}}$ is the settling delay of charge pump and the voltage regulator respectively. $T_{\text {Total }}$ dictates the DVTS loop bandwidth and governs the maximum rate of change of activity that can be supported. For DVTS loop convergence from point $\mathrm{A}$ to $\mathrm{C}$ in Fig. 4(a), value of $P$ is 18 and $Q$ is 12 . Using $T_{\mathrm{CNTRL}}=5 \mathrm{nS}, T_{\mathrm{INT}}^{\max }=125 \mathrm{nS}, T_{D, \mathrm{VBS}}=70 \mathrm{nS}$ and $T_{D, \mathrm{VDD}}=100 \mathrm{nS}$ the value of $T_{\text {Total }}=3.72 \mu \mathrm{S}$ [14], [15]. Whereas, from point $\mathrm{C}$ to $\mathrm{D} T_{\text {Total }}$ is $1.92 \mu \mathrm{S}$.

\section{B. Limits on the Size of the Load Circuit}

The achievable power savings $P_{S}$ by DVTS over DVS for a given activity and fix performance can be expressed as

$$
P_{S}=P_{\mathrm{DVS}}-\left(P_{\mathrm{PoP}}+P_{\mathrm{PM}}\right)
$$

where $P_{\mathrm{PoP}}$ is the power at $\mathrm{PoP}$ and $P_{\mathrm{PM}}$ is the total power overhead of the power monitor. By reducing block size $N$ of the load 


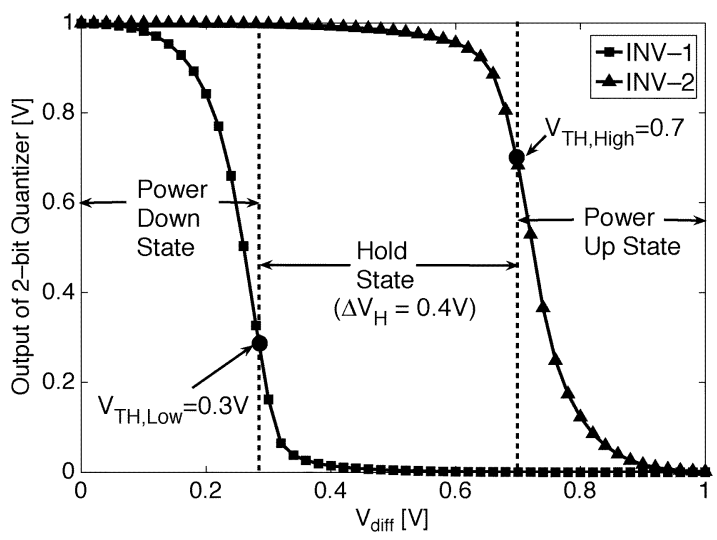

Fig. 9. Voltage transfer curve of 2-bit quantizer.

TABLE II

PARAMETER VALUES FOR TYPE-III LOAD CIRCUIT WITH REFERENCE TO NAND2 GATE

\begin{tabular}{|c|c|c|}
\hline Parameters & Description & Value \\
\hline$\Delta P_{H}$ & Power Threshold & $10 \mu \mathrm{W}$ \\
\hline $\mathrm{R}_{O N}$ & ON Resistance of Sleep Transistor & $4 \Omega$ \\
\hline $\mathrm{a}_{m}$ & Typical value for minimum activity & 0.001 \\
\hline $\mathrm{C}_{e f f}$ & Effective switch capacitance per gate & $7.6 \mathrm{fF}$ \\
\hline $\mathrm{V}_{D D}^{\min }$ & Minimum Supply Voltage & $0.8 \mathrm{~V}$ \\
\hline $\mathrm{f}_{\min }$ & Performance at $\mathrm{V}_{D D}^{\min }$ & $250 \mathrm{MHz}$ \\
\hline
\end{tabular}

circuit, the power savings reduce as $P_{\mathrm{PM}}$ stays constant while $P_{\mathrm{DVS}}$ and $P_{\mathrm{PoP}}$ reduces. Thus, for DVTS to achieve at least $S$ percent of power saving over DVS, the load circuit should have atleast $N_{\min }$ gates. For these gates $P_{\mathrm{PM}}$ has to be designed such that it is less than $S P_{\mathrm{DVS}}$ over range of activity of interest. Using $P_{\mathrm{DVS}}=\left[N \cdot C_{\mathrm{eff}} \cdot V_{\mathrm{DD}}^{2} \cdot a_{m} \cdot f+N \cdot V_{\mathrm{DD}} \cdot I_{\text {Leak }}\right]$, minimum block size, $N_{\min }$ for the load circuit is

$$
N_{\min }>\frac{P_{\mathrm{PM}}}{S \cdot\left[C_{\mathrm{eff}} \cdot V_{\mathrm{DD}}^{2} \cdot a_{m} \cdot f+V_{\mathrm{DD}} \cdot I_{\text {Leak }}\right]} .
$$

For Type-III circuit, the value of $S$ at $a=0.01 \& T=75^{\circ} \mathrm{C}$ is $21.6 \%$ (see Table I). Using the values from Table II and substituting them in (10), $N_{\min }$ is obtained to be $77 \mathrm{~K}$ gates. With scaling to DSM nodes, leakage power is larger which increases $S$ and thus reduce $N_{\min }$ whereas, switching power reduces which increases $N_{\min }$. Also, $P_{\mathrm{PM}}$ increases with scaling because at lower supply voltages op-amp burns more power to meet same dynamic range [16]. For low activity leakage power dominates total power and as leakage power increases with technology, $N_{\min }$ reduces. Thus, $N_{\min }$ scales along with technology for low activity load circuits. However, for high activity dynamic power dominates and it decreases with scaling which results in increase of $N_{\min }$ with scaling (see Table III). Note that (10) is a theoretical calculation of $N_{\min }$. In practice, $N_{\min }$ would be mainly driven by affordable voltage domains and micro-architecture choice [17].

\section{Minimum Activity Change Detectable}

Power monitor can track the changes in power which are above power threshold $\Delta P_{H}$. The minimum detectable change
TABLE III

EFFECT OF SCALING ON $N_{\text {min }}$ WITH VARYING $a$ FOR $S=20 \%$. UMC MODEL FILE FOR 90 and $65 \mathrm{~nm}$ AND PTM CARD FOR $45 \mathrm{~nm}$ ARE USED

\begin{tabular}{|c|c|c|c|}
\hline Activity(a) & $90 \mathrm{~nm}$ & $65 \mathrm{~nm}$ & $45 \mathrm{~nm}$ \\
\hline \hline $\mathrm{P}_{P M}$ & $200 \mu \mathrm{W}$ & $325 \mu \mathrm{W}$ & $480 \mu \mathrm{W}$ \\
\hline \hline 0.001 & $496 \mathrm{~K}$ & $101 \mathrm{~K}$ & $97 \mathrm{~K}$ \\
\hline 0.01 & $77 \mathrm{~K}$ & $74 \mathrm{~K}$ & $88 \mathrm{~K}$ \\
\hline 0.1 & $11 \mathrm{~K}$ & $20 \mathrm{~K}$ & $42 \mathrm{~K}$ \\
\hline
\end{tabular}

TABLE IV

SUMMARY OF DVTS TEST CHIP

\begin{tabular}{|c|c|}
\hline Process Technology & $0.35 \mu \mathrm{m}$ 2P4M CMOS Twin-well \\
\hline \multirow{3}{*}{ Power Supply } & Load Circuit: $\mathrm{V}_{D D}=0.8 \mathrm{~V}-3.3 \mathrm{~V}$ \\
\cline { 2 - 2 } & PM: $\mathrm{V}_{C C}=3.3 \mathrm{~V}$ \\
\cline { 2 - 2 } & $\mathrm{I} / \mathrm{O}: 3.3 \mathrm{~V}$ \\
\hline Operating Frequency & $4 \mathrm{MHz}$ to $25 \mathrm{MHz}$ \\
\hline \multirow{2}{*}{ Area } & Total: $700 \mu \mathrm{m}$ X $750 \mu \mathrm{m}$ \\
\cline { 2 - 2 } & PM: $200 \mu \mathrm{m} \mathrm{X} 250 \mu \mathrm{m}$ \\
\hline $\mathrm{V}_{B S}$ Range & $-0.5 \mathrm{~V}$ to $2.5 \mathrm{~V}$ \\
\hline \multirow{2}{*}{ Avg Power Consumed } & Load Circuit: $11 \mathrm{~mW} @ 3.3 \mathrm{~V}$ \\
\cline { 2 - 2 } & PM: $0.244 \mathrm{~mW} @ 3.3 \mathrm{~V}$ \\
\hline
\end{tabular}

in activity, $\Delta a$, can be detected if it brings a change in power greater than $\Delta P_{H}$. This can be expressed as

$$
\Delta P_{H} \leq \Delta a \cdot \frac{\partial P_{\mathrm{DVS}}}{\partial a}=\Delta a C_{\mathrm{eff}} V_{\mathrm{DD}, \min }^{2} f_{\mathrm{min}} N_{\mathrm{min}} .
$$

In practice, $\Delta a$ is a design specification for which $\Delta P_{H}$ is evaluated using (11). Using (11) and the values in Table III, if $\Delta a$ of 0.00011 is required to be detected around activity of 0.01 than the value of $\Delta P_{H}$ is $10 \mu \mathrm{W}$. Power monitor is engineered using this value of $\Delta P_{H}$ and (6).

\section{Technology Scalability of DVTS System}

The total leakage current in the load circuit is contributed by sub-threshold leakage, gate leakage and gate-induced drain leakage (GIDL). With technology scaling the gate leakage increases which is primarily managed by using high-K gate materials. Whereas, the sub-threshold leakage current still increases by $5 \times$ with each technology generation. DVTS yields maximum savings when the sub-threshold leakage dominates the total leakage current. Hence, DVTS can be beneficial in advance technology nodes. However, these savings depends on the effectiveness of reverse body bias (RBB) to control threshold voltage which is diminishing with every technology node. The scaling theory predicts that larger RBB will be required to cause enough change in threshold voltage. However, in practice RBB is still effective at 28,45 , and $65 \mathrm{~nm}$ technology by careful channel engineering [17]-[19].

The key challenge in scaling the power monitor to DSM nodes is the design of the integrating opamp. The opamp consumes majority of power to achieve required UGB and low input referred noise while meeting the required dynamic range. Techniques to improve dynamic range and intrinsic device gain in [16] and [20] can also be used for power monitor design. 


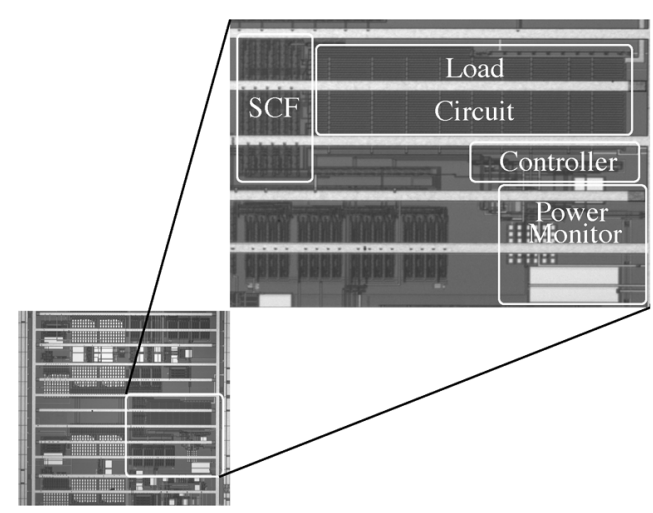

Fig. 10. Die micrograph.

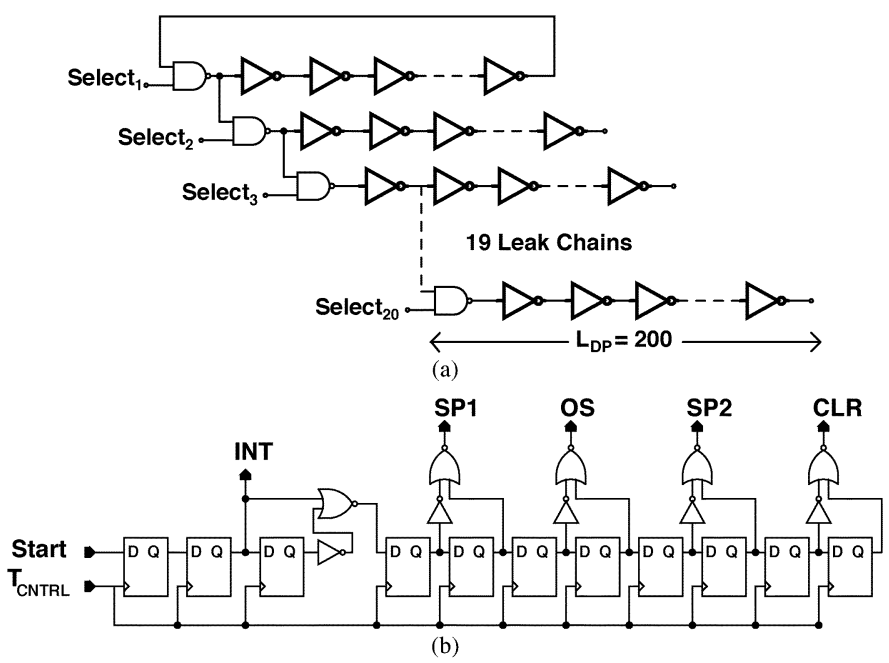

Fig. 11. (a) Load circuit. (b) CSG.

\section{TEST CHIP}

The test chip contains load circuit, controller, power monitor and scan flops as shown in Fig. 10. Scan flip-flops (SCF) selectively enables the inverter chain to control the activity factor from 0.05 to 1 . As the test chip is fabricated in a double well CMOS process, only nWell contacts were available and therefore, only pMOS body bias control is used to change the threshold voltage. Fig. 11(a) shows the load circuit consisting of 20 chains of inverters [21]. Select input of NAND gate enables or disables the respective chain. Out of 20 chains only the first chain is configured as an oscillator. In order to change resolution of power monitor $R_{\mathrm{INT}} C_{\mathrm{INT}}$ is made programmable by using R-bank for $R_{\mathrm{INT}}$. Fig. 11(b) shows circuit to generate control signal for power monitor. Table III list area and power consumption of load circuit and power monitor (PM).

\section{A. Measurement of Power Monitor Resolution}

Voltage resolution of power monitor is an important metric for the DVTS system. It is the minimum change at the virtual ground node which power monitor can resolve. The resolution of power monitor is mainly governed by integration time and

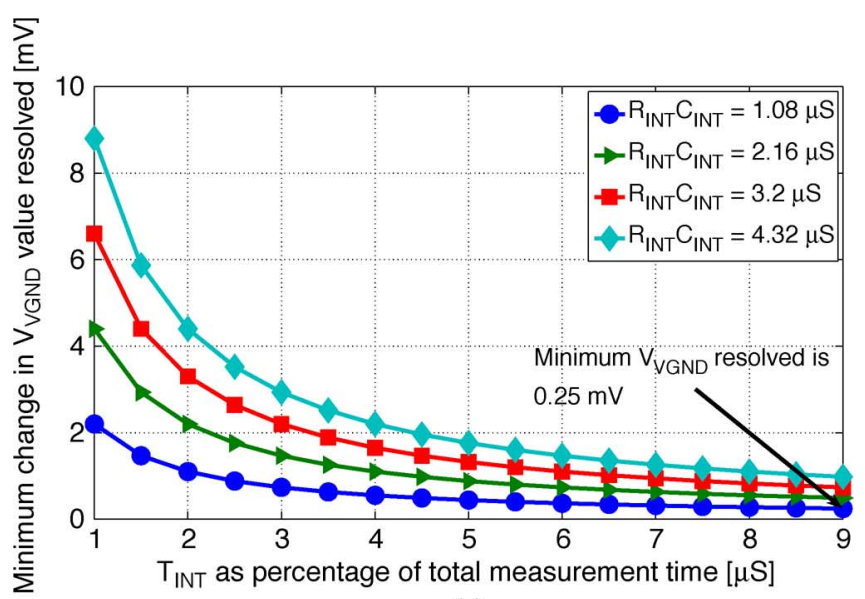

(a)

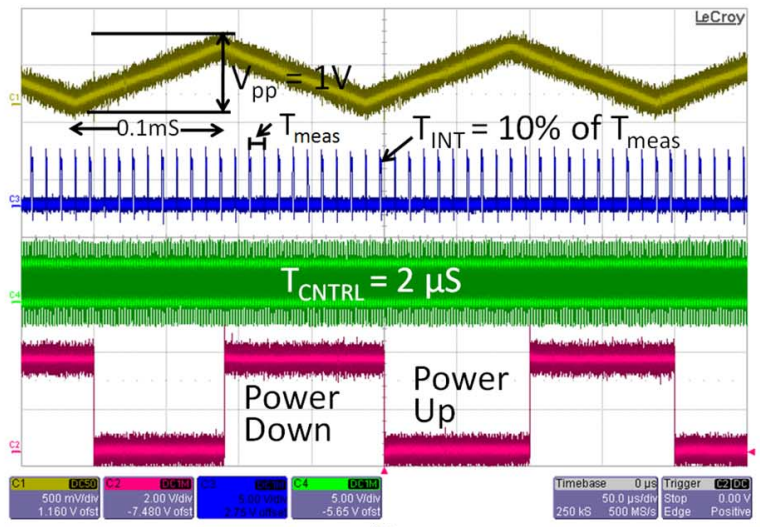

(b)

Fig. 12. (a) Measured resolution with varying $\mathrm{T}_{\mathrm{INT}}$. (b) CRO snap-shot of power monitor operation with $R_{\mathrm{INT}} C_{\mathrm{INT}}=1.08 \mu \mathrm{S}$. Output bits of power monitor Bit-0 and Bit-1 are multiplexed into 1 bit.

$R_{\mathrm{INT}} C_{\mathrm{INT}}$ time constant. Fig. 12(a) shows measured resolution of power monitor with different settings of $R_{\mathrm{INT}} C_{\mathrm{INT}}$. Best resolution is achieved for minimum available $R_{\mathrm{INT}} C_{\mathrm{INT}}$ and $T_{\mathrm{INT}}^{\max }$. Power monitor can resolve a minimum $250 \mu \mathrm{V}$ of variation in $V_{\mathrm{VGND}}$. For $T_{\mathrm{INT}}$ varying from $10 \%$ to $90 \%$ of $T_{\text {meas }}$, power monitor consumes an average power of $0.244 \mathrm{~mW}$. To measure resolution of power monitor, a triangular test signal is feed at virtual ground node. Power monitor samples test signal by $T_{\mathrm{INT}}$ as it ramps up and resolves it as power UP while when test signal ramps down power monitor resolves it as power going DOWN. As the frequency of test signal is reduced the change in $V_{\mathrm{VGND}}$ value between two consecutive $T_{\mathrm{INT}}$ reduces. Thus, for given $T_{\mathrm{INT}}$, resolution of power monitor is then defined by smallest frequency of test signal for which power monitor makes first error and decides HOLD state. As the test signal is triangular in nature the power monitor output should be either UP state or DOWN state. Power monitor indicates HOLD state only when it commits error due to failure in resolving its input. $T_{\text {meas }}$ is chosen such that after $T_{\mathrm{INT}}$ finishes, sufficient cycles of $T_{\mathrm{CNTRL}}$ are left to generate control signals. CRO snapshot in Fig. 12(b) shows test signal, integrate pulse and controller clock along with power monitor output. Note that if $T_{\text {meas }}$ is large and hold capacitors are not sufficiently sized than the charge loss due to $I_{\text {Leak }}$ degrades the resolution. 


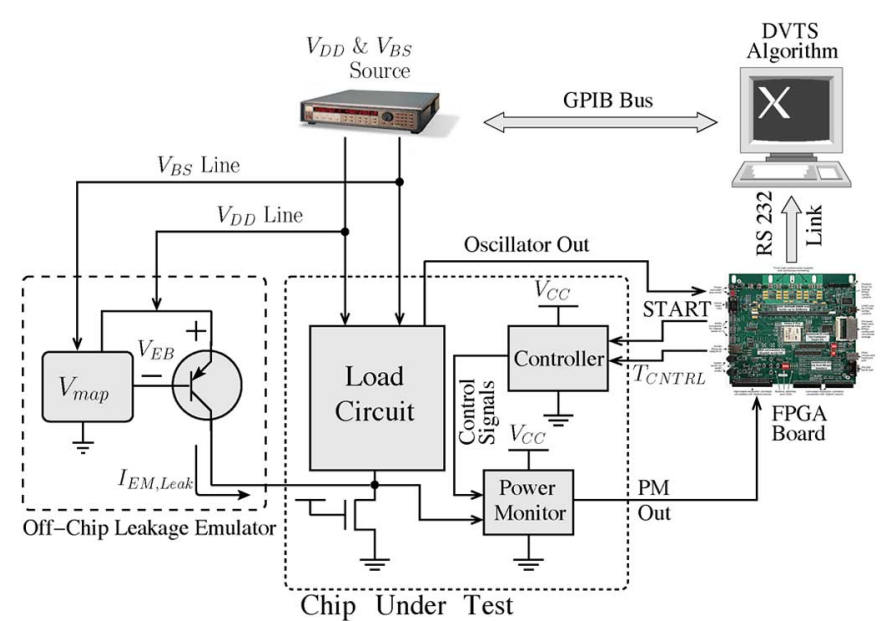

Fig. 13. DVTS test setup with leakage emulator. $T_{\mathrm{CNTRL}}$ of $67 \mathrm{nS}$ is fed from fixed signal source.

\section{B. DVTS Loop Convergence for Load Circuit Operating in Super-Threshold}

Fig. 13 shows the test setup for DVTS loop. To leverage the use of power monitor in advanced technology nodes, an off-chip leakage emulator is used to mimic sub-threshold leakage in 45 and $65 \mathrm{~nm}$ CMOS process using PTM files. Off the shelf opamps are used to generate $V_{\text {map }}$ such that the relation between $V_{E B}$ and $V_{B S}$ is similar to that between $V_{T H}$ and $V_{B S}$. DVTS algorithm runs off-chip on PC using MATLAB whereas rests of the components are on-chip. This test setup is sufficient to characterize power monitor and fine tune the DVTS algorithm. Adaptive $N$ bit counter to generate $T_{\mathrm{INT}}$ from $T_{\mathrm{CNTRL}}$ is implemented in FPGA and it is controlled over RS-232 link by the DVTS algorithm. As the load circuit is a ring oscillator, its performance can be monitored by monitoring its frequency of oscillation [21]. The performance monitor is implemented in FPGA. Two DC sources that power $V_{\mathrm{DD}}$ and $V_{B S}$ lines are controlled by DVTS algorithm over GPIB link. $V_{C C}$ and I/O ring power lines are kept constant at $3.3 \mathrm{~V}$ throughout the experiment. Fig. 14(a) shows the PoP for different activity after injecting emulated leakage current. DVTS algorithm is initiated at point-A [see Fig. 14(b)] to track PoP. Fig. 14(c) shows the stepping of $V_{\mathrm{DD}}$ and $V_{B S}$ line as the loop tracks PoP. The $\Delta V_{\mathrm{DD}}$ and $\Delta V_{B S}$ were chosen to be 50 and $100 \mathrm{mV}$, respectively. Point $\mathrm{B}$ in Fig. 14(a) is the DVS point located by the loop with $3 \%$ tolerance. The large continued transition from point $\mathrm{B}$ to $\mathrm{D}$ reflects poor sensitivity of delay with $V_{B S}$. Ideally, once the DVTS loop hits constant performance curve, it should directly locate PoP without searching for it. The total error in locating the DVS point, $\Delta V_{\mathrm{DVS}}$, is around $3 \cdot \Delta V_{\mathrm{DD}}$ due to three error sources namely, finite performance tolerance window, finite resolution of performance monitor and finite $\Delta V_{D D}$. Point $\mathrm{Q}$ is the ideal PoP [also shown in Fig. 14(a)] whereas, the DVTS loop settles to point $\mathrm{P}$ making finite error of $10 \mathrm{mV}$ in $V_{\mathrm{DD}}$ and 0.7 $\mathrm{V}$ in $V_{B S}$. Due to this error even though loop achieves power savings of $46.3 \%$, it loses possible power saving of around $7 \%$. Loop converges in around $18 \mathrm{~s}$ with present test setup. Convergence time is large mainly due to the sluggish DC sources used

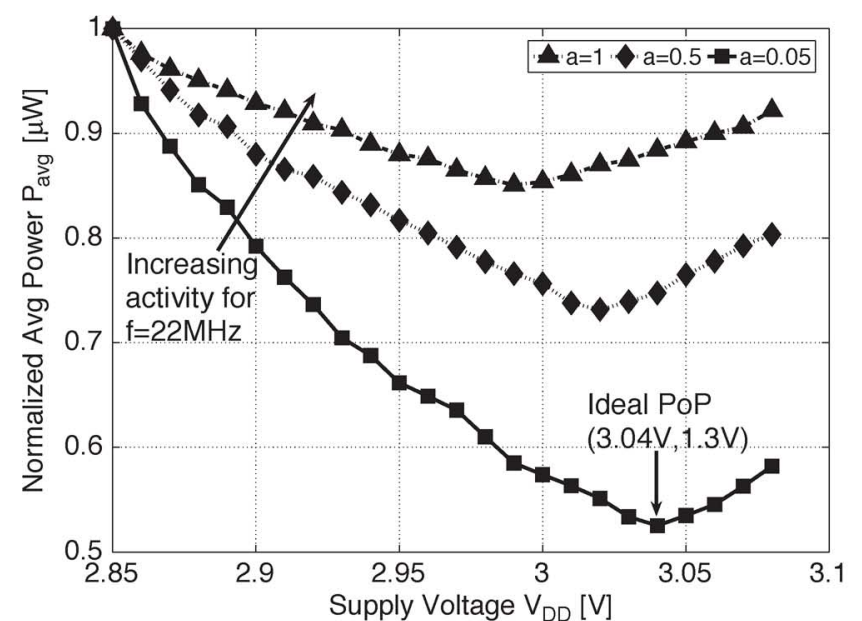

(a)

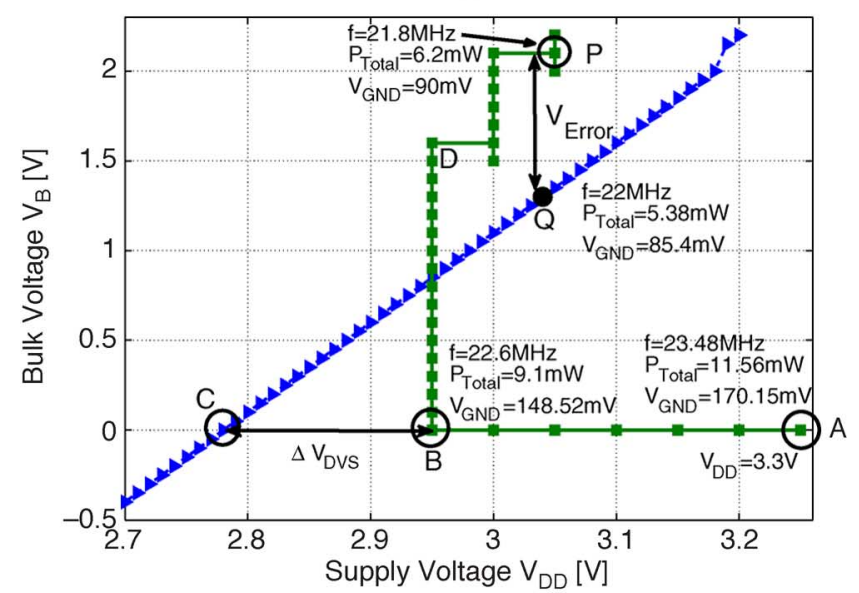

(b)

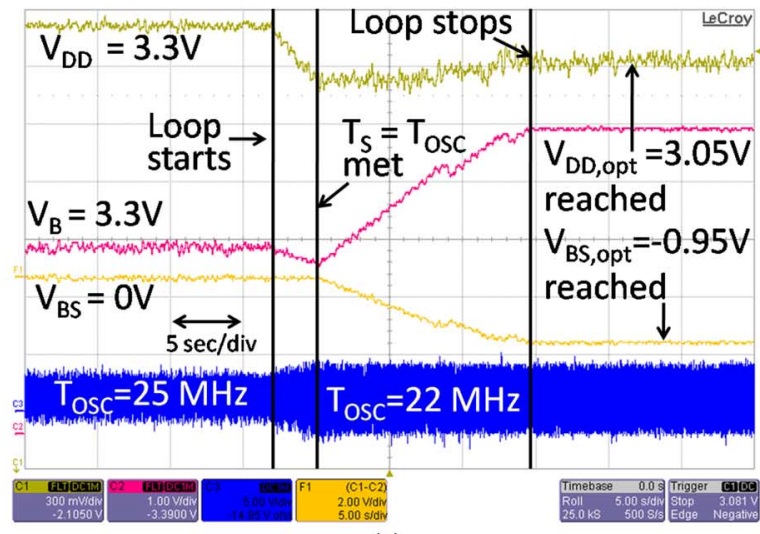

(c)

Fig. 14. (a) Total power of test chip after injecting leakage. (b) Contour followed by DVTS algorithm in $V_{\mathrm{DD}}-V_{B S}$ design space for performance of 22 MHz. (c) CRO snapshot during of DVTS operation and loop convergence.

for powering the supply and body voltages. DVTS algorithm locates and settles to $V_{\mathrm{DD} \text {,opt }}=3.05 \mathrm{~V}$ and $V_{B S \text {,opt }}=2.1 \mathrm{~V}$ as shown in Fig. 14(c).

\section{DVTS Loop Convergence for Load Circuit Operating Near Sub-Threshold Region}

As the loop approaches PoP, the power monitor is required to resolve finer changes in virtual ground voltage. These changes are even finer at lower supply voltage. Due to large delay of DC 


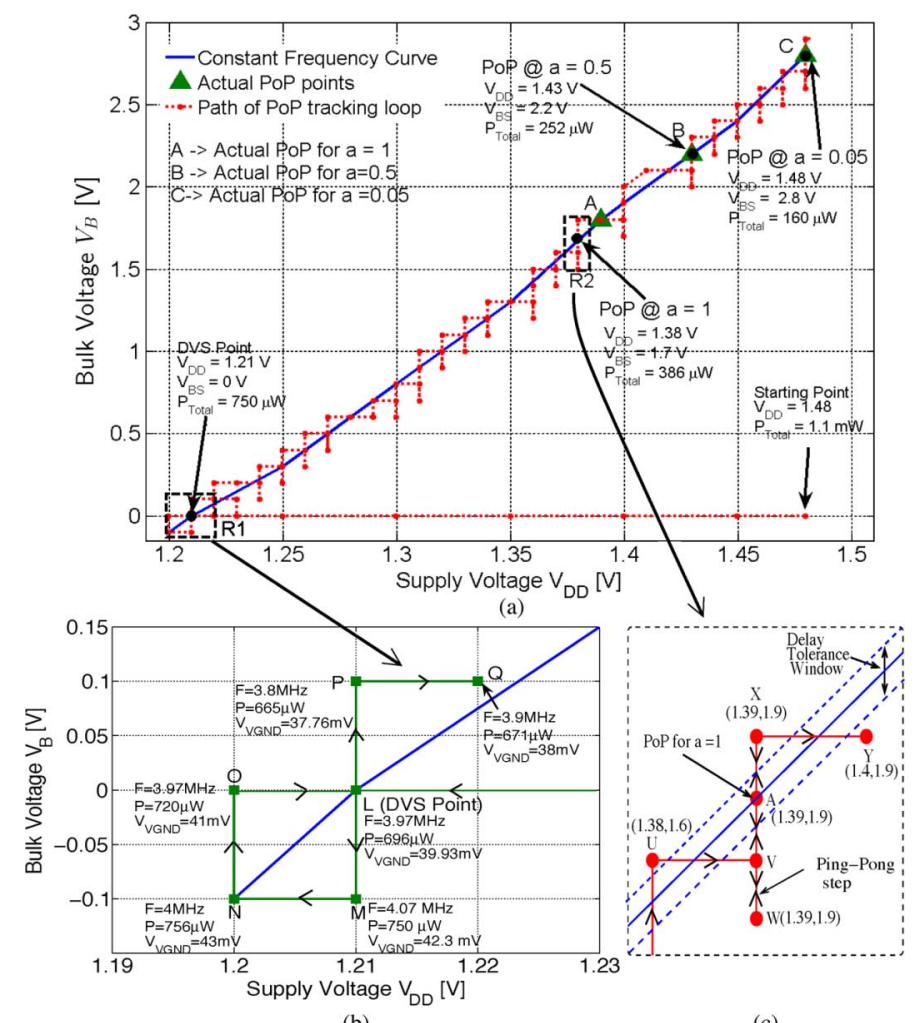

(b)

(c)

Fig. 15. (a) DVTS loop convergence for near sub-threshold operation $\left(T_{S}=\right.$ $250 \mathrm{n}$ ). (b) departure from and arrival at DVS point. (c) Departure from PoP.

sources the measurement time $T_{\text {meas }}$ is large which induces charge loss from $\mathrm{C} 2$ due to leakage current. This degrades the resolution of power monitor which causes DVTS loop to commit errors at some $\left\{V_{\mathrm{DD}}, V_{B S}\right\}$ steps as it approaches PoP. To enable the study of convergence of algorithm (see Fig. 3) and principle of power monitor at lower supply voltages, power monitor output in Fig. 13 was replaced by volt-meter which was controlled over the GPIB link. To make load circuit operate near sub-threshold, it was powered with $V_{\mathrm{DD}}$ of $1.5 \mathrm{~V}$. Value chosen for $\Delta V_{\mathrm{DD}}$ and $\Delta V_{B S}$ is 10 and $100 \mathrm{mV}$, respectively. The loop starts with average power consumption of $1.1 \mathrm{~mW}$ and $T_{S}$ of $4 \mathrm{MHz}$ [see Fig. 15(a)]. Point A, B, and C are the ideal PoP for activity of $1,0.5$, and 0.05 , respectively. As shown in Fig. 15(b), after reaching DVS point L, DVTS algorithm goes around path L-M-N-O because at lower $V_{\mathrm{DD}}$ the sensitivity of delay to $\left\{V_{\mathrm{DD}}, V_{B S}\right\}$ are comparable. Converging to PoP of $\mathrm{a}=1$ at point A saves $65 \%$ of power from starting point and $48.5 \%$ from DVS point. Once loop settles at point A (PoP for $a=1$ ) the activity of load circuit is reduced to 0.5 and the DVTS loop re-initiates itself. Departure of DVTS algorithm from PoP point A towards new PoP at point $\mathrm{B}$ is shown in Fig. 15(c). The dotted lines in the Fig. 15(c) is the tolerance window within which the algorithm locates PoP. Ping-pong step V-W occurs due to reasons explained in Section II.

\section{Comparison With Other State-of-the Art Techniques}

Table V shows a comparison of different power management techniques with this work. For the approach in [7] and [9] and
TABLE V

State-OF-THE-ARt Power MANAgEMENT TeChniques

\begin{tabular}{|l|l|l|l|l|l|l|}
\hline Features & {$[\mathbf{8}]$} & {$[\mathbf{2 2}]$} & {$[\mathbf{6}]$} & {$[\mathbf{3}]$} & {$[\mathbf{9 ]}$} & $\begin{array}{l}\text { This } \\
\text { work }\end{array}$ \\
\hline $\begin{array}{l}\text { Activity } \\
\text { Tracking }\end{array}$ & Periodic & External & External & External & Auto & Auto \\
\hline $\begin{array}{l}\text { Sensitivity } \\
\text { to PVT }\end{array}$ & No & Yes & No & Yes & No & No \\
\hline $\begin{array}{l}\text { Power } \\
\text { monitor }\end{array}$ & Direct & Replica & Direct & Replica & Direct & Direct \\
\hline $\begin{array}{l}\text { Area(mm } \\
\text { Overhead }\end{array}$ & - & $\begin{array}{l}0.25 \\
\text { @90nm }\end{array}$ & $\begin{array}{l}0.05 \\
\text { @65nm }\end{array}$ & - & $\begin{array}{l}2.56 \\
\text { @90nm }\end{array}$ & $\begin{array}{l}0.05 \\
\text { @ 350nm }\end{array}$ \\
\hline $\begin{array}{l}\text { Overhead } \\
\text { Scaling }\end{array}$ & Yes & Yes & Yes & Yes & No & No \\
\hline
\end{tabular}

this work activity can be tracked automatically. For rest of the approaches external input is required to indicate change in activity and reinitiate the control loop. Also the overhead of this work (and [9]) does not scale up with increasing size of the load circuit. Compared with the replica approach [3], the key benefits of proposed scheme is ability to track PoP with activity. Even if external activity information is available, the replica approach can track PoP in discrete steps of activity which limits achievable power saving. Further, replica approach is sensitive to region of operation of load circuit. Also, replica based approach is heuristic approach where control loop is operated to locate desired $I_{S W} / I_{\text {Leak }}$ while present work locates PoP in true sense.

\section{CONCLUSION}

A DVTS algorithm that can locate $\left\{V_{\mathrm{DD} \text {,opt }}, V_{B S \text {,opt }}\right\}$ with varying activity is studied under simulation framework and validated with a test chip. Timing overhead of DVTS system and size of load circuit are analyzed as they limit the application of DVTS. The tracking performance of the algorithm can be improved by reducing the timing overhead. It can be inferred that for technology node with larger leakage currents, DVTS is more beneficial over DVS.

The key contribution of paper is the in-situ power monitor. The dynamic range of power monitor is a crucial parameter which determines the range of $V_{\mathrm{DD}}$ scaling. Digitally assisted techniques can be used to improve the dynamic range. The accuracy of power monitor is also critical as it directly impacts the power savings possible by DVTS control. To improve the accuracy, techniques to cancel the input offset voltage of difference amplifier can be employed. To avoid resolution degradation due to leakage current the analog storage needs to be replace with a robust digital solution. Also, with technology scaling the design of integrator can become challenging and hence, alternate integrator topologies (e.g., VCO) has to be researched.

\section{APPENDIX A}

\section{ACTIVITy DEPENDENT NoISE IN POWER MONITOR}

The activity of the load circuit can be approximated by stationary random variable with a Poisson distribution with mean and variance of $\bar{a}$ [7]. With this approximation, the total current in Fig. 5 is

$$
i_{\text {Total }}(t)=a(t) \cdot C_{S W} \cdot V_{\mathrm{DD}} \cdot f+i_{\text {Leak }} \cdot
$$


Due to fluctuation in the activity the total current varies with mean $\overline{i_{\text {avg }}}$ and variance $\sigma_{i}^{2}$ which are given by

$$
\overline{i_{\mathrm{avg}}}=\bar{a} C_{S W} V_{\mathrm{DD}} f+i_{\text {Leak }} \quad \sigma_{i}^{2}=\bar{a} C_{S W}^{2} V_{\mathrm{DD}}^{2} f^{2} .
$$

Using (12) and (1), the mean and variance of $V_{\mathrm{INT}}$ is

$$
\begin{aligned}
\overline{V_{\mathrm{INT}}} & =K_{v} R_{\mathrm{ON}} T_{\mathrm{INT}} \overline{i_{\mathrm{avg}}} \\
\& \quad \sigma_{V_{\mathrm{INT}}}^{2} & =K_{v}^{2}\left[R_{\mathrm{ON}}^{2} \sigma_{i}^{2}\left(\frac{T_{\mathrm{INT}}}{f}\right)\right] .
\end{aligned}
$$

The equation above assumes that the cycle to cycle activity variations are independent. Substituting the equations above in (4) the deviation in the power measurement $P_{a}$ is

$$
P_{a}=V_{\mathrm{DD}}^{2} \cdot C_{S W} \sqrt{\frac{\bar{a} f}{T_{\mathrm{INT}}}} .
$$

\section{ACKNOWLEDGMENT}

The authors would like to thank the anonymous reviewers for their helpful suggestions. They would also like to thank Prof. N. Bhat for his support with chip tapeout.

\section{REFERENCES}

[1] T. Burd, T. Pering, A. Stratakos, and R. Brodersen, "A Dynamic voltage scaled microprocessor system," IEEE J. Solid-State Circuits, vol. 35 , no. 11 , pp. 1571-1580, Nov. 2000.

[2] K. Nose and T. Sakurai, "Optimization of VDD and VTH for lowpower and high speed applications," in Proc. Asia South Pacific Des. Autom. Conf., 2000, pp. 469-474.

[3] M. Nomura, Y. Ikenaga, K. Takeda, Y. Nakazawa, Y. Aimoto, and Y. Hagihara, "Delay and power monitoring schemes for minimizing power consumption by means of supply and threshold voltage control in active and standby modes," IEEE J. Solid-State Circuits, vol. 41, no. 4, pp. 805-814, Apr. 2006.

[4] J. Kao, M. Miyazaki, and A. Chandrakasan, "A 175-MV multiply-accumulate unit using an adaptive supply voltage and body bias architecture," IEEE J. Solid-State Circuits, vol. 37, no. 11, pp. 1545-1554, Nov. 2002.

[5] T. Kuroda, K. Suzuki, S. Mita, T. Fujita, F. Yamane, F. Sano, A. Chiba, Y. Watanabe, K. Matsuda, T. Maeda, T. Sakurai, and T. Furuyama, "Variable supply-voltage scheme for low-power high-speed CMOS digital design," IEEE J. Solid-State Circuits, vol. 33, no. 3, pp. 454-462, Mar. 1998.

[6] Y. Ramadass and A. Chandrakasan, "Minimum energy tracking loop with embedded DC-DC converter enabling ultra-low-voltage operating down to $250 \mathrm{mV}$ in $65 \mathrm{~nm}$ CMOS," IEEE J. Solid-State Circuits, vol. 43, no. 1, pp. 256-265, Jan. 2008.

[7] S. Naffziger, "Sampling chip activity for real time power estimation," U.S. Patent 20090259869 , Oct. 15, 2009.

[8] R. Jotwani, S. Sundaram, S. Kosonocky, A. Schaefer, V. Andrade, A. Novak, and S. Naffziger, "An x86-64 core implemented in $32 \mathrm{~nm}$ SOI CMOS," IEEE J. Solid-State Circuits, vol. 46, no. 12, pp. 162-172, Dec. 2010.

[9] R. McGowen, C. Poirier, C. Bostak, J. Ignowski, M. Millican, W. Parks, and S. Naffziger, "Power and temperature control on a 90-nm Itanium family processor," IEEE J. Solid-State Circuits, vol. 41, no. 1, pp. 229-237, Jan. 2006.

[10] M. Elgebaly and M. Sachdev, "Variation-aware adaptive voltage scaling system," IEEE Trans. Very Large Scale Integr. (VLSI) Syst., vol. 15 , no. 5, pp. 560-571, May 2007.

[11] S. Lee and T. Sakurai, "Run-time power control scheme using software feedback loop for low-power real-time applications," in Proc. AsiaPacific Des. Autom. Conf, 2000, pp. 381-386.

[12] N. Mehta, G. Naik, and B. Amrutur, "In-Situ power monitoring scheme and its apllication in dynamic voltage and threshold scaling for digital CMOS integrated circuits," in Proc. IEEE Int. Symp. Low Power Electron. Des., 2010, pp. 259-264.

[13] C. Enz and G. Temes, "Circuit techniques for reducing the effects of op-amp imperfections: Autozeroing, correlated double sampling, and chopper stabilization," Proc. IEEE, vol. 84, no. 11, pp. 1584-1614, Nov. 1996.

[14] W. Kim, M. Gupta, G. Wei, and D. Brooks, "System level analysis of fast, per-core DVFS using on-chip switching regulators," in Proc. Int. Symp. High-Perform. Comput. Arch., 2008, pp. 123-134.

[15] D. Levacq, M. Takamiya, and T. Sakurai, "Backgate bias accelerator for 10 ns-order sleep-to-active modes transition time," in Proc. IEEE Asian Solid-State Circuits Conf., Nov. 2007, pp. 2390-2395.

[16] M. Park, "A 4th order continuous-time ADC with VCO-based integrator and quantizer," Ph.D. dissertation, Dept. Elect. Eng. Comput. Sci., Massachusetts Institute of Technology (MIT), Cambridge, MA, 2009.

[17] G. Gammie, A. Wang, M. Chau, S. Gururajarao, R. Pitts, F. Jumel, S. Engel, P. Royannez, R. Lagerquist, H. Mair, J. Vaccani, G. Baldwin, K. Heragu, R. Mandal, M. Clinton, D. Arden, and U. Ko, "A 45 nm $3.5 \mathrm{G}$ baseband-and-multimedia application processor using adaptive body-bias and ultra-low-power techniques," in Dig. Tech. Papers IEEE Int. Solid-State Circuits Conf. (ISSCC), 2008, pp. 258-611.

[18] D. Mansur and S. Shumarayev, "Introducing $28 \mathrm{~nm}$ stratix V FPGAs: Built for bandwidth," presented at the Hot Chips Symp., Palo Alto, CA, 2010.

[19] M. Togo, T. Fukai, Y. Nakahara, S. Koyama, M. Makabe, E. Hasegawa, M. Nagase, T. Matsuda, K. Sakamoto, S. Fujiwara, Y. Goto, T. Yamamoto, T. Mogami, M. Ikeda, Y. Yamagata, and K. Imai, "Poweraware $65 \mathrm{~nm}$ node CMOS technology using variable VDD and backbias control with reliability consideration for back-bias mode," in Dig. Tech. Papers Symp. VLSI Technol., 2004, pp. 88-89.

[20] Y. Chiu, B. Nikolic, and P. Gray, "Scaling of analog-to-digital converters into ultra-deep-submicron CMOS," in Proc. Custom Integr. Circuits Conf., 2005, pp. 375-382.

[21] A. Wang and A. Chandrakasan, "Optimal supply and threshold scaling for subthreshold CMOS circuits," in Proc. IEEE Comput. Soc. Annu. Symp. VLSI, 2002, pp. 5-9.

[22] Y. Ikenaga, M. Nomura, Y. Nakazawa, and Y. Hagihara, "A circuit for determining the optimal supply voltage to minimize energy consumption in LSI circuit operations," IEEE J. Solid-State Circuits, vol. 43, no. 4, pp. 911-918, Apr. 2008.

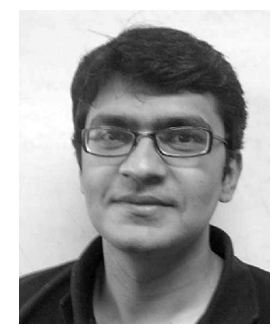

Nandish Mehta (S'11) received the B.E. degree in electronics and communication engineering from the Dharmsinh Desai University, Nadiad, India, in 2005.

From 2005 to 2007, he worked with Indian Space Research Organization. Since 2007, he has been working as a full time researcher with the Indian Institute of Science, Bangalore, India. His research interests include adaptive power efficient SoCs voltage converters and on-chip measurement techniques.

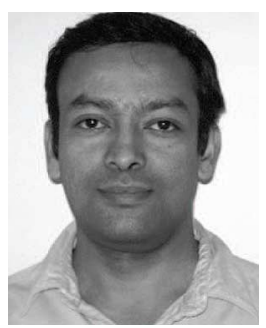

circuits and systems.
Bharadwaj Amrutur (M'08) received the B.Tech. degree in computer science and engineering from Indian Institute of Technology, Bombay, India, in 1990 and the M.S. and Ph.D. degrees in electrical engineering from Stanford University, Palo Alto, CA, in 1994 and 1999, respectively.

He has worked at Bell Labs, Agilent Labs, and Greenfield Networks. He is currently an Assistant Professor with the Department of Electrical Communication Engineering, Indian Institute of Science, Bangalore, India, working in the areas of VLSI 